\title{
Medium and Long-term Sales Forecast of Hydrogen Fuel Cell Vehicles (HFCV) Based on System Dynamics and Discrete Selection Model
}

\author{
Liqing Shao ${ }^{1, \mathrm{a}}$, Xudong $\mathrm{Li}^{1}$ and Shouxi Wu${ }^{1}$ \\ ${ }^{1}$ CATARC (Tianjin) Automotive Information Consulting Co., Ltd.,Tianjin, China
}

\begin{abstract}
Developing hydrogen fuel cell vehicle is an important direction for the transformation and upgrading of automobile industry, but its current development is not clear yet. This paper uses a discrete selection model and a system dynamics model to analyze the key factors affecting the sales of hydrogen fuel cell vehicles, and analyze the vehicle purchasing behavior of consumers. The medium and long-term sales volume of hydrogen fuel cell vehicles is predicted in various typical scenarios. The forecast results show that the government subsidies and changes in the number of hydrogen refueling stations have a great influence on the medium and long-term sales of hydrogen fuel cells. The energy prices and the breakthrough in the core technologies of key components also have a certain influence on future sales.
\end{abstract}

\section{Introduction}

In China, the HFCV industry is not mature, and historical data deficiency prevents sales prediction with data-driven methods. Against such a background, the System Dynamics method is adopted in this paper to, at a macro level, analyze the sales process of HFCVs systematically, determine the main influence factors, and define the influence mechanism. In addition, the method is also used in combination with the discrete choice model to, at the micro level, describe the purchasing behaviors of customers, carry out comprehensive correction based on predicted data of experts, foreign data and data on other vehicle types, establish a sales evolution model of HFCVs in multiple scenarios and analyze typical policy scenarios to predict HFCV sales.

\section{Introduction Basic Theoretical Method}

\subsection{System Dynamics}

System Dynamics (SD) is a methodology proposed by Forrester, a renowned American professor, after years of research to solve complicated social problems in a systematic way. With the method, concrete problems are abstracted into causal relationships with a computer platform, exploring the influence mechanism of realistic problems qualitatively and quantitatively ${ }^{[1]}$.

\subsection{Discrete Choice Model}

The discrete choice model is a market study research technology that is both very effective and practical ${ }^{[2]}$. The model, based on experimental design, measures the purchasing behaviors of customers by simulating the market competition environment of the products and services to be studied. This helps to understand how the customers make choices under different conditions of products, service attribute levels and prices ${ }^{[3]}$.

\section{Creation of Model}

\subsection{System Analysis and Establishment of the Causal Diagram}

The energy used differentiates HFCVs from the traditional oil-fueled vehicles. As a result, the cost and utilization mode of HFCVs - the main factors influencing the sales-are different from those of the oil-fueled ones ${ }^{[4]}$.

The causal relationship of the sales of HFCV passenger vehicles is as shown in Fig. 1. HFCV bus, HFCV medium truck and HFCV heavy truck share the same causal relationship with the HFCV passenger vehicle. 


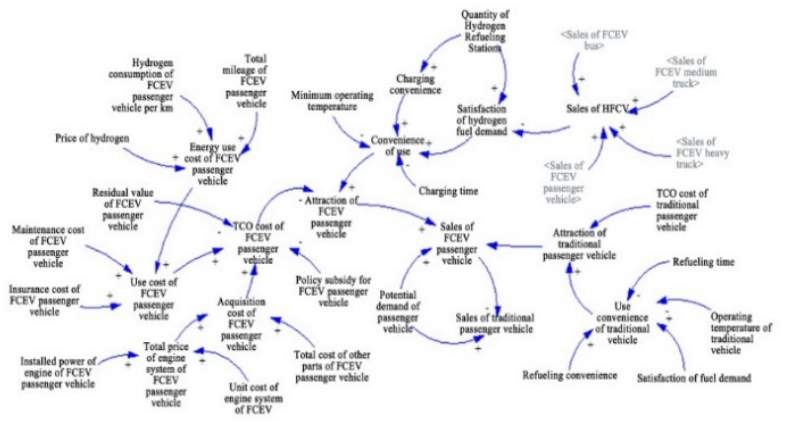

Figure 1. Causal Diagram of the HFCV Sales

\subsection{Creation of System Dynamics Model}

The system flow of the prediction model of HFCV sales is created. The system creates a model with the number of HFCVs in operation as the state variable and the sales of HFCVs as the rate variable. The discrete choice model is selected after the relational expression to calculate the sales of HFCVs is determined.

When a customer is purchasing a vehicle, the probability of selecting an HFCV is ${ }^{[5]}$ :

$$
P(\text { hydrogen fuel })=\frac{1}{1+e^{\theta\left(u_{c j u a n}-u_{q i n g}\right)}}
$$

Where, $u_{c \text { juan }}$ and $u_{\text {qing }}$ refer to the utility value of a traditional vehicle and an HFCV, respectively; $\theta$ refers to a parameter.

When the total demand of the market is known, the sales of the HFCVs is:

$$
\frac{1}{1+\mathrm{e}^{\theta *\left(\mathrm{u}_{\text {chuan }} \mathrm{u}_{\text {qing }}\right)}} \text { Sales of HFCVs }=\text { potential demand } *
$$

In the model, the variables of the utility of a traditional vehicle and an HFCV are the attraction of the vehicles. The size of the utility depends on TCO and the use convenience of the vehicles. Their relationship is shown in the equation below ${ }^{[6]}$ :

$$
\text { Utility }=\frac{\alpha}{\text { Vehicle TCO cost }}+\beta * \text { use convenience(3) }
$$

Based on the causal relationship between the variables, the computational formula of TCO and the use convenience of a vehicle is as follows:

Use convenience $=$ charging convenience + satisfaction of fuel demand $-0.04 *$ charging time $0.01 *$

minimum operating temperature vehicle $\mathrm{TCO}$ cost $=$ energy use cost + insurance expense + vehicle maintenance cost + vehicle acquisition cost vehicle residual value - government subsidy (4)

Different vehicle types differ significantly from each other in terms of costs and relevant parameters. Therefore, HFCVs are divided into four subsystems according to the vehicle type: passenger vehicles, buses, medium trucks and heavy trucks. Parameters are set with existing data to reflect the features and development trends of different vehicle types.

(1) The main equation for passenger vehicles: attraction of FCEV passenger vehicle $=0.64 *$

HFCV use convenience $+0.74 *$ 50

TCO cost of FCEV passenger vehicle

attraction of traditional passenger vehicle $=0.64 *$ use convenience of traditional vehicle $+0.56 *$

$\frac{50}{\text { TCO cost of traditional passenger vehicle }}$

Sales of HFCV passenger vehicle $=$ potential demand of passenger vehicle *

$\frac{1}{1+\mathrm{e}^{7.5 *\left(\mathrm{u}_{\text {chuan }} 1^{\left.-\mathrm{u}_{\text {qing } 1}\right)}\right.}}$

(2) The main equation for coaches:

Attraction of HFCV bus $=0.78 *$

HFCV use convenience $+1.05 *$

$\frac{400}{\text { TCO cost of HECV passenger vehicle }}$

Attraction of traditional bus $=0.53 *$ use convenience of traditional bus $+0.62 *$

$\frac{400}{\text { TCO cost of traditional bus }}$

Sales of HFCV bus $=$ potential demand of bus * $\frac{1}{1+\mathrm{e}^{5.3 *\left(\mathrm{u}_{\text {chuan }} 2^{\left.-\mathrm{u}_{\text {qing }}\right)}\right.}}$

(3) The main equation for medium trucks: Attraction of HFCV medium truck $=0.63 *$ HFCV use convenience $+0.64 *$ $\frac{160}{\text { TCO cost of HFCV medium truck }}$ use convenience of traditional medium truck + $0.52 * \frac{160}{\text { TCO cost of traditional medium truck }}$

(4) The main equation for heavy trucks: Attraction of HFCV heavy truck $=0.63 *$ FCEV use convenience $+0.65 *$

$\frac{480}{\text { TCO cost of HFCV heavy truck }}$

Attraction of traditional heavy truck $=0.54 *$ use convenience of traditional heavy truck $+0.283 *$ 480

TCO cost of traditional heavy truck

Sales of HFCV heavy truck $=$ potential demand of heavy truck *

$\frac{1}{1+\mathrm{e}^{6.5 *\left(\mathrm{u}_{\text {chuan } 4}-\mathrm{u}_{\text {qing } 4}\right)}}$

To determine some of the parameters in the relational expression, existing historical data are employed for fit and simulation analysis during the model creation.

\subsection{Results and Correction of Model Simulation}

Given the fact that there are few data on HFCV sales in China, foreign data, predicted results of experts and sales of other vehicle types are adopted to correct and adjust the simulation results of the model. 


\section{Sales Prediction by Scenario}

(1) Benchmark Scenario

In the benchmark scenario, the number of hydrogen refueling stations will increase steadily from 300 in 2025 to 1,000 in 2030. The cost of the hydrogen fuel cell engine system will drop to RMB $1,500 / \mathrm{kW}$ by 2030 . The price of hydrogen will decrease slowly to RMB 40/kg by 2030 . There will still be subsidies which, however, are to be reduced by $20 \%$ each year. The simulation is carried out for the period from 2020 to 2030 . The specific data are as shown in Table 1.

Due to the rapid increase in the number of hydrogen refueling stations and the decrease of TCO year by year, the sales will increase rapidly from 2023 to 2025 and is expected to reach 66,000 in 2025. However, as the sales increase, the demand for hydrogen refueling stations increases rapidly by 2026 with the number of HFCVs in operation grows quickly. The TCO will decrease slowly due to the declining subsidy, which leads to a slowdown in the later sales growth. By 2030, the total sales will be 603,000 .

From the perspective of sales structure, before 2027, commercial vehicles contribute most to the development of HFCVs. It is estimated that after 2027, the sales of passenger vehicles will be 10,000 and more, and by 2030, the sales of the four types of vehicles will be more than 100,000 .

(2) Scenario for Adjustment of the Number of Hydrogen Refueling Stations

Modify the number of hydrogen refueling stations, reduce the growth rate of hydrogen refueling stations, and build 300 hydrogen refueling stations by 2030 . Compared with the baseline scenario, the trend of sales volume is basically the same year by year, but the total sales volume is obviously reduced. By 2030, it still cannot reach the annual sales volume of 100,000.

(3) Scenario of hydrogen price adjustment

Provided that the hydrogen price is maintained the same at RMB 60/kg since 2020, analyze the influence of hydrogen price against the sales volume. Compared with the baseline scenario, use cost of vehicles increases, total sales volume decreases obviously, and the sales volume will be up to 377,000 by 2030 .

(4) Scenario of government subsidy adjustment

Assume that the government subsidy is cancelled since 2024. Compared with the baseline scenario, the vehicle acquisition cost increases and the total sales volume decreases significantly, which also makes the sales volume fluctuate greatly and be more susceptible to interference from other factors. By 2030, the sales volume will be up to 500,000.

(5) Scenario of technical progress adjustment

The HFCV technology is still evolving rapidly. Provided that the cost of hydrogen fuel cell engine system declines rapidly and falls to RMB 1050/kW by 2030, the TCO cost of vehicle will be decreased accordingly. Compared with the baseline scenario, the engine system cost will be greatly reduced from 2025 to 2030, which will reduce the TCO cost and increase the sales volume faster. By 2030, the total sales volume will be up to 1 million.

\section{Conclusion}

The following conclusions are got based on the prediction results of a model in different scenarios:

(1) The number of hydrogen refueling stations greatly affects the HFCV sales. Only a large penetration of hydrogen refueling stations can drive the marketization of HFCVs. To solve the construction and operation cost of hydrogen refueling stations, the government should reinforce investment, encourage investment in enterprises, and jointly develop the HFCV sales and construction of hydrogen refueling stations.

(2) The energy price greatly affects the use cost of a vehicle, especially for consumers with high use frequency and large energy consumption. A higher energy price imposes a greater economic burden. At present, the technology of hydrogen production needs to be further broken through to realize large-scale hydrogen production from renewable energy.

(3) Government subsidies can stimulate distinctly the sales of HFCVs. Before the government subsidies are canceled, an effective alternative policy shall be introduced to ensure the sustainable development of the HFCV industry.

(4) Breakthroughs in core technologies and independent R\&D for key parts are of primary importance to cut vehicle costs and improve system performance, facilitating the long-term development of the HFCV industry. Therefore, the government shall provide effective assistance and support for enterprises in the capital, technology, talents and other aspects.

\section{Here are some examples:}

1. Li L, Simonovic S P. System dynamics model for predicting floods from snoemelt in North American prairie watersheds. Hydroligical Processes, 2002,16(13):2645 2666

2. Nie Chong, Jia Shenghua. Research on the theoretical basis and evolution of discrete choice models [J].Quantitative \& Technical Economics, 2005,12(11):151-159

3. Dong Xiaolan and Shen Hao, Application of Discrete Selection Model in Market Research [J], Journal of Communication University of China (Natural Science), 2002,9(4):4-42

4. Jiang Ruibin and Tan Ligang, Development and Challenges of Electric Vehicles [J], Mechanical Engineer, 2009, (2): 23-25

5. McFadden D.Economic choices [J].American Economic Re-view, 2001,91(3):351-378

6. Tu Xinshu, Wang Chunfeng and $\mathrm{Ba}$ Shusong, Research on Portfolio Utility Problem [J], the Journal of Quantitative \& Technical Economics, 2002,(5):3740 